\title{
Polypyrrole-Doped Conductive Self-Healing Composite Hydrogels with High Toughness and Stretchability
}

\author{
Lingling Zhao ${ }^{1}$, Xin $\mathrm{Li}^{2}$, Yan $\mathrm{Li}^{1}$, Xuemiao Wang ${ }^{1}$, Wu Yang ${ }^{1}$, Jie Ren ${ }^{1 *}$ \\ ${ }^{1}$ Chemistry \& Chemical Engineering College, Northwest Normal University, Key Lab of \\ Bioelectrochemistry \& Environmental Analysis of Gansu, Key Lab of Polymer Materials of Ministry of \\ Education of Ecological Environment, Lanzhou, 730070, PR China \\ ${ }^{2}$ The High School Attached to Northwest Normal University, Lanzhou, 730070, PR China
}

The polymerization of pyrrole occurred in a $250-\mathrm{mL}$ three-necked flask equipped with a reflux condenser. It was placed on an ice-water bath with a magnetic stirring bar to keep the reactor solution mixed. A platinum anode and a graphite cathode with a distance between them about $3 \mathrm{~cm}$ were used to produce GDEP. Initially, $30 \mathrm{~mL}$ pyrrole, $20 \mathrm{~mL}$ of $2 \mathrm{~g} \cdot \mathrm{L}^{-1} \mathrm{Na}_{2} \mathrm{SO}_{4}$ solution, and $0.06 \mathrm{~g}$ sodium dodecyl benzenesulfonate (SDBS) were added into the three-necked flask and stirred for $30 \mathrm{~min}$ at room temperature. Then, glowdischarge was carried out with an applied voltage of $600 \mathrm{~V}$ and a current of 60 to $70 \mathrm{~mA}$. The black polypyrrole was produced and deposited on the platinum anode slowly. The polypyrrole was washed with distilled water several times and dried under a vacuum at $60{ }^{\circ} \mathrm{C}$ until constant weight.

*Corresponding author: E-mail: jieren@ @wnu.edu.cn 